\title{
Improvement of heavy flavor production in a multiphase transport model updated with modern nuclear parton distribution functions
}

\author{
L. Zheng, ${ }^{1,2, *}$ C. Zhang $\odot,{ }^{2}$ S. S. Shi, ${ }^{2, \dagger}$ and Z. W. Lin ${ }^{2,3, *}$ \\ ${ }^{1}$ School of Mathematics and Physics, China University of Geosciences (Wuhan), Wuhan 430074, China \\ ${ }^{2}$ Key Laboratory of Quark and Lepton Physics (MOE) and Institute of Particle Physics, Central China Normal University, \\ Wuhan 430079, China \\ ${ }^{3}$ Department of Physics, East Carolina University, Greenville, North Carolina 27858, USA
}

(Received 16 September 2019; revised manuscript received 19 January 2020; accepted 19 February 2020; published 9 March 2020)

\begin{abstract}
Recently we updated a multiphase transport (AMPT) model with modern parton distribution functions of nuclei (nPDFs). Here we study open charm production in the updated AMPT model and compare to the experimental data from $p p$ and $A A$ collisions over a wide range of collision energies. Besides the update of nPDFs, we removed the transverse momentum cutoff on initial heavy quark productions and also included the resultant heavy flavor cross section into the total minijet cross section in the initial condition as described by the HIJING model. We show that the AMPT model with these updates provides a much better description of the yields and transverse momentum spectra of various open charm hadrons in comparison with the experimental data. This lays the foundation for further heavy flavor studies within the transport model approach.
\end{abstract}

DOI: 10.1103/PhysRevC.101.034905

\section{INTRODUCTION}

In high energy hadronic collisions, heavy flavor production provides us a powerful tool to study quantum chromodynamics (QCD) [1]. The initial production of heavy flavor quarks is calculable with the perturbative QCD (pQCD) method due to the relatively large value of the heavy quark mass. In heavy ion physics, heavy quarks also play an important role because their masses are typically larger than the temperatures achieved in the produced quark-gluon plasma (QGP). Therefore, they are predominantly produced in the initial hard scatterings between the two nuclei on a timescale shorter than the formation time of the QGP. As a result, heavy quarks can experience almost the full evolution of the deconfined nuclear medium and are thus sensitive to early dynamics of the collision system [2,3].

Recently, it was realized that the strong electromagnetic fields in the initial state of heavy ion collisions may significantly affect the heavy quark directed flow $v_{1}$ and result in a larger charm $v_{1}$ than lighter particles [4-6]. In addition, heavy quarks or hadrons are also expected to interact with the QGP or hadronic medium through elastic or inelastic processes during their propagation in the dense matter. This would lead to the suppression of heavy hadron yields at high transverse momentum often represented by the nuclear modification factor $R_{A A}$ and anisotropic flows of heavy hadrons such as elliptic flow $v_{2}$. These observables can be used to extract the transport

\footnotetext{
*zhengliang@cug.edu.cn

†shiss@mail.ccnu.edu.cn

*linz@ecu.edu
}

properties of the QGP matter such as the drag and diffusion coefficients [7-9]. For example, a large suppression in $R_{A A}$ and/or a substantially nonzero $v_{2}$ for open heavy particles indicates that heavy quarks experience significant interactions with the bulk medium.

Multiple theoretical frameworks have been developed to describe heavy flavor productions in high energy collisions. The fixed flavor number scheme (FFNS) [10] is the simplest scheme for the treatment of heavy flavors in the pQCD theory. Next-to-leading order (NLO) calculations are available for this approach, while the gluon fragmentation to heavy flavor hadrons is not included. Other implementations have been developed on the basis of the FFNS method. Results from the general-mass variable-flavor-number scheme (GMVFNS) approach $[11,12]$ as an extension to FFNS generally agree well with both the $p p$ and $p \mathrm{~A}$ data in a wide rapidity range. The fixed order plus next-to-leading logarithms (FONLL) formalism [13] is another widely used pQCD method that matches the massive FFNS cross section with the massless VFNS. This approach usually can reasonably describe the open charm experimental data, whereas its central value often under-predicts the data.

In addition, medium induced effects can be included with models based on the heavy quark transport or pQCD calculations of the parton energy loss. In the high transverse momentum $\left(p_{\mathrm{T}}\right)$ region, models $[14,15]$ that include both collisional and radiative energy loss of heavy quarks usually provide a fair description of the $R_{A A}$ from central to peripheral collisions. On the other hand, the evolution of low- $p_{\mathrm{T}}$ heavy quarks in the bulk medium is similar to Brownian motion and can thus be studied with transport approaches based on the Langevin or Boltzmann equation, which have been 
implemented in models such as POWLANG [16], TAMU [17], DUKE [18], BAMPS [19], LBT [20], MC@HQ+EPOS [21], PHSD [22], and CATANIA [23,24].

A multiphase transport (AMPT) model [25] is a useful tool to study the bulk medium through the microscopic dynamical processes of the evolving system. The ZPC component solves the Boltzmann equation for two-body scatterings via the parton cascade approach [26]. As a self-contained event generator, the AMPT model provides a unified framework to explore the medium evolution with different flavors including the event-by-event fluctuation and conservation of conserved charges. For example, the initial production of heavy quarks is modeled together with that of the light quarks, and therefore the conservation of quantities such as energy, momentum, net baryon number, and net charm number is guaranteed in the AMPT initial condition of each event. Transport model studies of the dense matter, including heavy flavor studies with the AMPT model [27,28], help us to understand the QGP evolution and the transition from the nonequilibration stage to the hydrodynamic stage [29-32]. In particular, systematic comparisons of model predictions with the measured heavy flavor $R_{A A}$ and $v_{2}[33,34]$ allow us to determine the relevant QGP medium transport coefficients such as the spatial diffusion constant $D_{s}$, the drag coefficient $\eta_{D}$, and the momentum transport coefficients $\kappa_{L}, \kappa_{T}, \hat{q}$.

Recently we updated the AMPT model with an improved quark coalescence process [35] and modern nuclear parton distribution functions (nPDFs) [36]. In this work, we will use the improved AMPT model to address open charm production and then compare the model results with the experimental data. The rest of the paper is organized as follows. In Sec. II we discuss the physics changes we have made for open heavy flavor productions in the AMPT model. We then calculate the yield and $p_{\mathrm{T}}$ spectra of various open charm hadrons in comparison with the experimental data in $p p$ and $A A$ collisions in Secs. III and IV, respectively. After discussions in Sec. V, we summarize in Sec. VI.

\section{DESCRIPTIONS OF HEAVY FLAVORS IN THE UPDATED AMPT MODEL}

In the AMPT model, the initial production of heavy quarks $(Q)$ is handled by the HIJING two-component model [37]. It includes pair productions $(q+\bar{q} \rightarrow Q+\bar{Q}, g+g \rightarrow Q+\bar{Q})$ and gluon splitting $(g \rightarrow Q+\bar{Q})$. The gluon splitting process is implemented with a parton shower method similar to that in general purpose Monte Carlo event generators [38,39], which includes the leading-log resummation of the multiple parton emission phenomenon [40]. However, the flavor excitation processes $(q+Q \rightarrow q+Q, g+Q \rightarrow g+Q)$ are not included. This is partly because the HIJING model only deals with closed string objects created by minijet productions, but a flavor excitation process usually delivers a single heavy quark jet in the final state. The pair production cross section for heavy quarks in pQCD at leading order can be expressed as

$$
\frac{d \sigma^{Q \bar{Q}}}{d p_{\mathrm{T}}^{2} d y_{1} d y_{2}}=K \sum_{a, b} x_{1} f_{a}\left(x_{1}, \mu_{F}^{2}\right) x_{2} f_{b}\left(x_{2}, \mu_{F}^{2}\right) \frac{d \sigma^{a b \rightarrow Q \bar{Q}}}{d \hat{t}}
$$

In the above, $y_{1}$ and $y_{2}$ are the rapidities of the two produced partons, the $K$ factor aims to account for higher-order corrections of heavy quark productions, $a$ and $b$ refer to the types of interacting partons in the initial state, $x$ denotes the nucleon momentum fraction taken by the interacting parton, $\mu_{F}$ represents the factorization scale, $f_{a}$ represents the parton distribution function of parton type $a$ in a (free or bound) nucleon, and $\sigma^{a b \rightarrow Q \bar{Q}}$ is the cross section for parton types $a$ and $b$ to produce the heavy quark pair. The $K$ factor $K=2.5$ is used for both light and heavy flavor productions, as done in our previous study of light flavor observables after the update of nPDFs [36]. For the charm quark production we take $\mu_{F}^{2}=$ $2\left(p_{\perp}^{2}+m_{c}^{2}\right)$, where $p_{\perp}$ is the transverse momentum transfer, and we take the charm quark mass as $m_{c}=1.3 \mathrm{GeV} / c^{2}$ in this study.

The calculation of hard scatterings in AMPT is implemented within the HIJING two-component model, where each hard parton is generated with a minimum transverse momentum cut, $p_{0}$, to regulate the total minijet production cross section. The differential minijet cross section at leading order has the same form as Eq. (1):

$$
\frac{d \sigma^{c d}}{d p_{\mathrm{T}}^{2} d y_{1} d y_{2}}=K \sum_{a, b} x_{1} f_{a}\left(x_{1}, \mu_{F}^{2}\right) x_{2} f_{b}\left(x_{2}, \mu_{F}^{2}\right) \frac{d \sigma^{a b \rightarrow c d}}{d \hat{t}}
$$

where $\sigma^{a b \rightarrow c d}$ is the cross section for parton types $a$ and $b$ to produce a pair of minijets $c$ and $d$. Then the total minijet cross section can be written as

$$
\sigma_{\mathrm{jet}}=\sum_{c, d} \frac{1}{1+\delta_{c d}} \int_{p_{0}^{2}}^{s / 4} d p_{\mathrm{T}}^{2} d y_{1} d y_{2} \frac{d \sigma^{c d}}{d p_{\mathrm{T}}^{2} d y_{1} d y_{2}} .
$$

This minijet transverse momentum cutoff $p_{0}$ and the soft interaction cross section $\sigma_{\text {soft }}$ are the two key parameters in the HIJING two-component model: they control the elastic, inelastic, and total cross sections of $p p$ and $p \bar{p}$ collisions $[36,37,41,42]$. Note that there is an extra factor of $1 / 2$ for final states with identical partons, such as $g+g \rightarrow g+g$ for minijet gluon productions, in the above equation. In contrast, the original HIJING model applies the factor of $1 / 2$ to all light flavor minijet production processes [41], and that leads to a slightly smaller total $\sigma_{\text {jet }}$ than Eq. (3) (at the same $p_{0}$ ).

In the HIJING model as well as the previous AMPT model (denoted as "old AMPT") before our most recent updates as done in Ref. [36] and this study, the minijet cross section of Eq. (3) does not include the cross section of heavy flavors such as charm and bottom quarks as given by Eq. (1). As a result, $\sigma_{\text {jet }}$, which is used in the eikonal formalism for the total, elastic, and inelastic cross sections [37,41], represents the cross section of light flavor $(u / d / s)$ minijets. Then in the actual generation of minijets, heavy flavor minijets are still being generated and their fraction is calculated by the ratio of the heavy flavor cross section over the total minijet cross section that includes both light and heavy flavors, where the same minimum transverse momentum cut $p_{0}$ is used in calculating $\sigma^{Q \bar{Q}}$ as in Eq. (3) for $\sigma_{\text {jet }}$.

The above approach has two issues. First, for selfconsistency the heavy flavor cross sections need to be included in the total minijet cross section in the two-component model. 
Second, the heavy quark cross sections can be calculated with $\mathrm{pQCD}$ without any minimum transverse momentum requirement, and the large heavy quark mass (compared to $\Lambda_{\mathrm{QCD}}$ ) naturally regulates the heavy quark total cross section. Therefore, we make significant changes to the descriptions of heavy flavors in the AMPT model by removing the $p_{0}$ cut for the heavy quark production cross sections and then including them in the total minijet cross section. These changes can be illustrated by the following modified formula for the new minijet cross section:

$$
\begin{aligned}
\sigma_{\text {jet }}= & \sum_{c, d} \frac{1}{1+\delta_{c d}} \int_{p_{0}^{2}}^{s / 4} d p_{\mathrm{T}}^{2} d y_{1} d y_{2} \frac{d \sigma_{\text {light }}^{c d}}{d p_{\mathrm{T}}^{2} d y_{1} d y_{2}} \\
& +\sum_{c, d} \int_{0}^{s / 4} d p_{\mathrm{T}}^{2} d y_{1} d y_{2} \frac{d \sigma_{\text {heavy }}^{c d}}{d p_{\mathrm{T}}^{2} d y_{1} d y_{2}},
\end{aligned}
$$

where the first term represents the total cross section of light flavor $(u / d / s / g)$ minijets and the second term represents that of heavy flavor minijets including charm and bottom flavors.

Naively one may expect that including the heavy quark production cross sections in the total minijet cross section will have negligible effects because heavy quarks are very rare. However, in a two-component model such as HIJING the light flavor $(u / d / s / g)$ minijets require a minimum $p_{0}$ while we think the heavy flavor minijets should not. Therefore when the $p_{0}$ value is high, which is especially the case for both HIJING 2.0 [42] and the updated AMPT model [36] that use newer PDFs, the charm production cross section may be a significant fraction of the total minijet cross section for $A A$ collisions at high energies. Note that in the AMPT model updated with new nPDFs we have related the $p_{0}$ value for central $A A$ collisions at high energies to that for $p p$ collisions with a nuclear scaling of $p_{0}$ [36]. That leads to a larger $p_{0}$ in $A A$ collisions and thus suppresses light flavor minijet productions at high energies, while the initial heavy flavor production is not affected.

The event averaged $c \bar{c}$ yield as a function of the collision energy is shown in Fig. 1. Results without a heavy quark channel in the total minijet cross section are represented by the dashed curves while the solid curves show the charm quark pair numbers after heavy flavor channels are included as in Eq. (4). The inclusion of heavy quark cross section slightly increases the charm quark yield at high energies in $p p$ collisions. At low energies, the difference between these two curves becomes negligible as expected due to the small heavy flavor cross section. In $A A$ collisions, the impact of including the heavy flavor cross section in $\sigma_{\text {jet }}$ becomes more significant, up to a factor of 2 at very high energies. We also see a faster increase of the charm quark yield with the colliding energy in $A A$ collisions than that in $p p$ collisions at high energies. This is because the ratio of the charm quark yield in $A A$ collisions over that in $p p$ collisions at the same energy is roughly the number of binary collisions, which is proportional to the inelastic nucleon-nucleon scattering cross section that increases with the colliding energy.

We expect the string melting version of the AMPT model [43] (instead of the default version of AMPT) to be applicable in describing the dense matter at high energies when a QGP is believed to be formed in the early stage of the collisions.

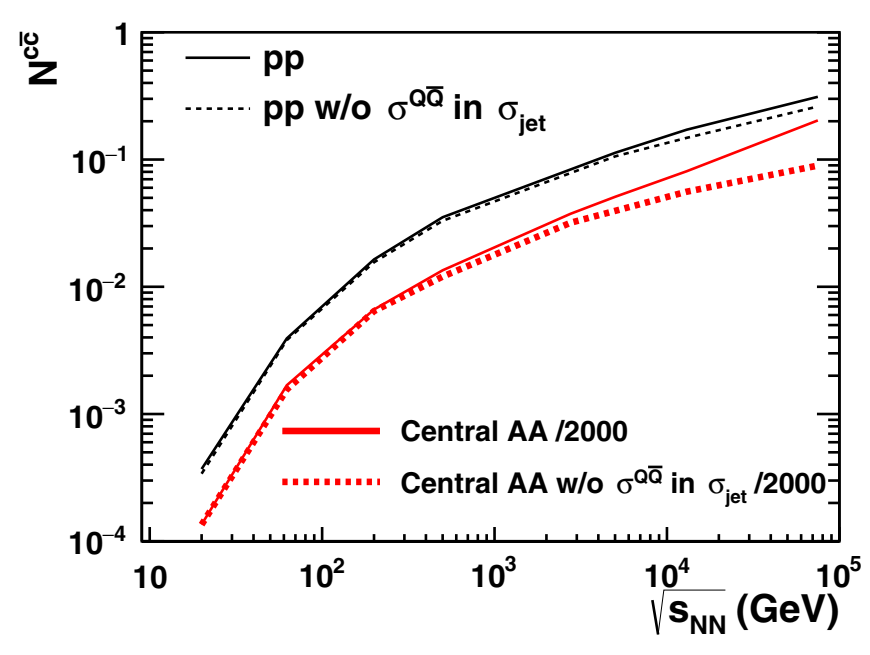

FIG. 1. Effect of including heavy flavor cross sections into the minijet cross section on the yield of charm-anticharm quark pairs from the AMPT model for $p p$ and central $A A$ collisions $(\mathrm{Au}+\mathrm{Au}$ at or below $200 \mathrm{GeV}$ and $\mathrm{Pb}+\mathrm{Pb}$ above $200 \mathrm{GeV}$ ).

Therefore we use the string melting version throughout this study. The hadronization of the partonic matter is accomplished by a spatial quark coalescence model [35] after partons stop interacting. The open heavy flavor hadron species formed by quark coalescence includes the following charm and bottom hadrons at all possible charge states (plus the corresponding anti-particles when applicable): $D, D^{*}, D_{s}, D_{s}^{*}$, $\Lambda_{c}, \Sigma_{c}, \Xi_{c}, \Xi_{c}^{\prime}, \Xi_{c c}, \Omega_{c}, \Omega_{c c}, \Omega_{c c c}$, as well as $B, B^{*}, B_{s}, B_{s}^{*}$, $B_{c}, B_{c}^{*}, \Lambda_{b}, \Sigma_{b}, \Xi_{b}, \Xi_{b}^{\prime}, \Xi_{b c}, \Xi_{b c}^{\prime}, \Xi_{b b}, \Omega_{b}, \Omega_{b c}, \Omega_{b c}^{\prime}, \Omega_{b b}$, $\Omega_{b b c}$, and $\Omega_{b b b}$. The hadron species are determined by the flavor combination of the two or three coalescing (anti)quarks. In addition, for a pseudoscalar meson and a vector meson with the same flavor combination, our previous approach is to form the meson to which the invariant mass of the coalescing quark and antiquark is closer [25]. However, we find that the resultant vector- to pseudoscalar-meson ratios such as the $K^{*} / K$ ratio and the $D^{*} / D$ ratio are often far away from the experimental data [44]. Therefore, we change the previous approach and now set the ratio of each type of vector to pseudoscalar meson in the quark coalescence model: 0.30 for primordial $\rho / \pi, 0.50$ for primordial $K^{*} / K$, and 1.0 for primordial $D^{*} / D$ or $B^{*} / B$. For example, for all the flavor combinations that could form either $D$ or $D^{*}$ mesons, we order them in terms of the excess mass (i.e., the difference between the two-quark invariant mass and the sum of two quarks' masses) and assign the half with lower excess masses to form $D$ and the half with higher excess masses to form $D^{*}$. The above values are chosen to roughly reproduce the overall magnitudes of the final vector- to pseudoscalar-meson ratios observed in $p p$ collisions of various energies. Note that the above values determine the ratios of the primordial (i.e., right after quark coalescence) meson multiplicities in each event, not the ratios in the final state that often include effects from resonance decays and hadronic rescatterings.

In the new quark coalescence model for AMPT [35], the overall relative probability of a quark to form a baryon instead of a meson is determined by the $r_{B M}$ parameter, which is 
assumed to be independent of the colliding energy and collision system. Generally, there would be no antibaryon formation if this parameter is 0 but almost no meson formation if it goes to infinity. In the updated AMPT model [36] used for this work, the $r_{B M}$ value for light flavor $(u / d / s)$ hadrons is set to 0.53 . On the other hand, the $r_{B M}$ value for heavy flavor hadrons is set to 1.0, because using the light flavor value would lead to too few charm baryons (by a factor of $\approx 4$ ) compared to the experimental data in $p p$ or $A A$ collisions. More specifically, we determined this heavy flavor $r_{B M}$ value according to the $\Lambda_{c}$ measurements in $p p$ collisions at the Large Hadron Collider (LHC) [as shown in Fig. 5(b)]. In principle, the $r_{B M}$ value for charm hadrons is related to properties such as the number and masses of available charm baryon states versus charm meson states, and the higher $r_{B M}$ value for charm is consistent with the assumption that relative to light flavors there are more charm baryon states than charm meson states [45].

Heavy quark productions directly depend on the PDFs as shown in Eq. (1). In addition, for $A A$ collisions $f$ in Eqs. (1) and (2) represents the parton PDFs in the nucleus instead of those in a free nucleon and thus contains the nuclear shadowing functions. Therefore we expect that our recent updates to the AMPT model [36] by including the newer parton distribution functions CTEQ6.1M [46] of free nucleons and the modern impact parameter-dependent EPS09s [47] nuclear shadowing functions should improve its descriptions of heavy flavor productions.

\section{OPEN CHARM RESULTS FOR $p p$ COLLISIONS}

We now use the string melting version of the updated AMPT model [36], which already includes the heavy flavor improvements as detailed in the previous section, to study the open charm production in $p p$ collisions in this section as well as $A A$ collisions in the next section. Like the study on light flavors [36], we set the Lund string fragmentation parameters to $a=0.8, b=0.4 \mathrm{GeV}^{-2}$ for $p p$ collisions and

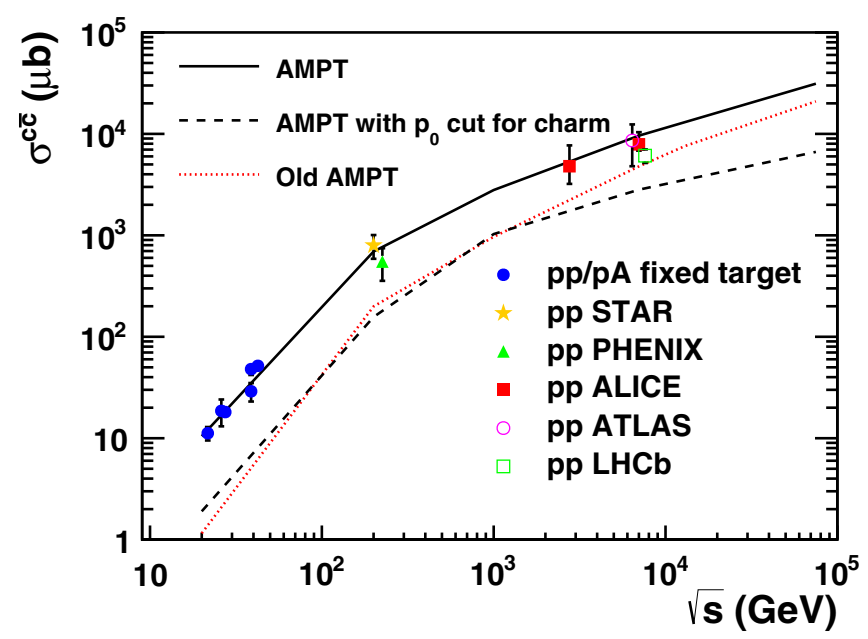

FIG. 2. Total cross sections of charm-anticharm quark pairs from AMPT for $p p$ collisions in comparison with the world data [49-55] as functions of the colliding energy. $a=0.8, b=0.15 \mathrm{GeV}^{-2}$ for the central $\mathrm{Au}+\mathrm{Au}$ collisions at Relativistic Heavy Ion Collider (RHIC) energies and $\mathrm{Pb}+$ $\mathrm{Pb}$ collisions at LHC energies. In addition, we set the parton elastic scattering cross section to $\sigma_{p}=3 \mathrm{mb}$ and the hadron cascade cutoff time to $30 \mathrm{fm} / c$. The old AMPT results are often provided for comparisons, and there we use the AMPT version v2.26t9 with the same parameters as in an earlier study [48]. Unless otherwise specified, the yield of each charm meson species represents the average of the particles and the corresponding antiparticles.

The total $c \bar{c}$ pair cross section varying with $\sqrt{s}$ for $p p$ collisions from the AMPT model is shown in Fig. 2 compared with the available world data. The data points from PHENIX, ATLAS, and LHCb collaborations are slightly shifted horizontally. We see that the updated AMPT model with our recent modifications (solid line) can provide a good description of the charm quark cross section in $p p$ collisions in a wide energy range. On the other hand, the old AMPT model significantly under-estimates the charm cross section, especially at low energies. The dashed line shows the results when the charm
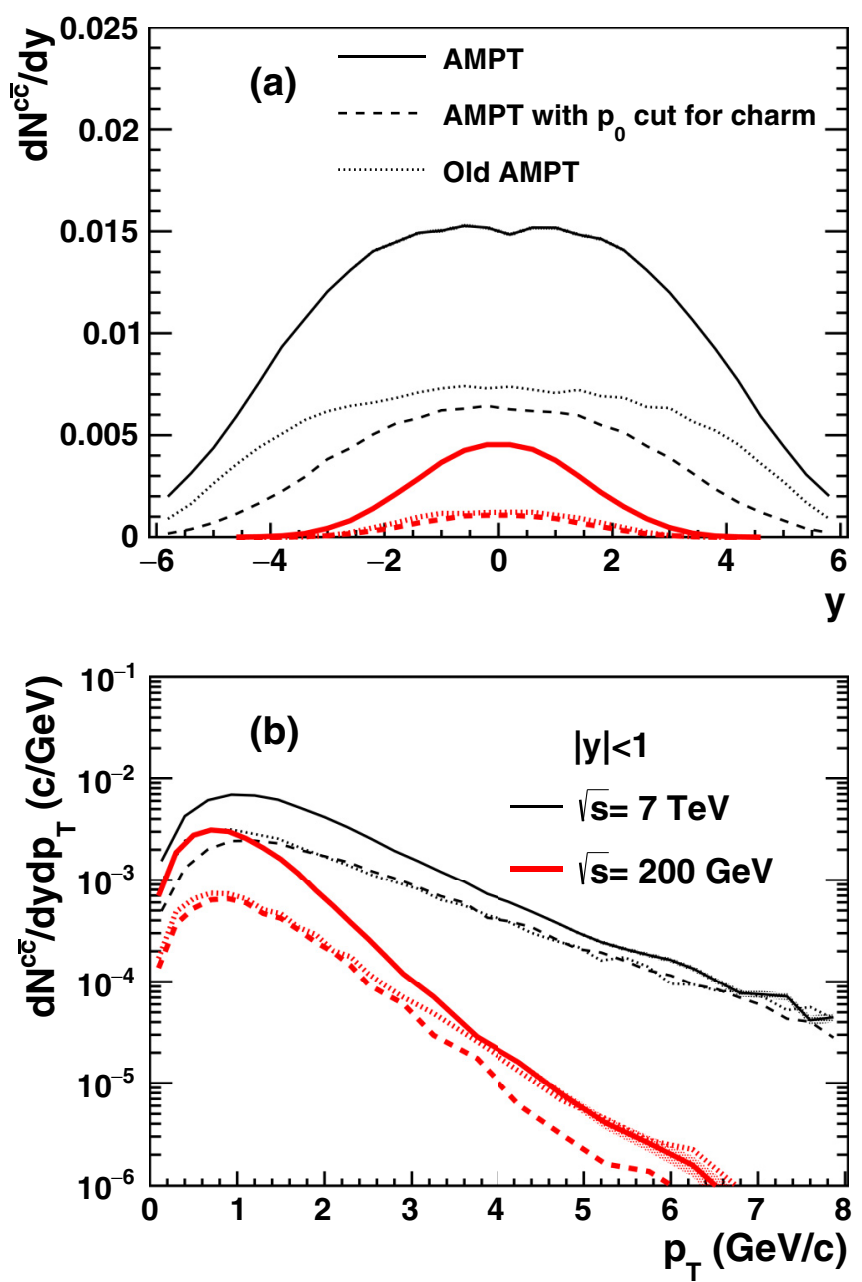

FIG. 3. (a) Rapidity distributions and (b) transverse momentum spectra of charm quarks in $p p$ collisions at $\sqrt{s}=200 \mathrm{GeV}$ (thick) and $\sqrt{s}=7 \mathrm{TeV}$ (thin) from the AMPT model. The shaded band represents statistical errors. 

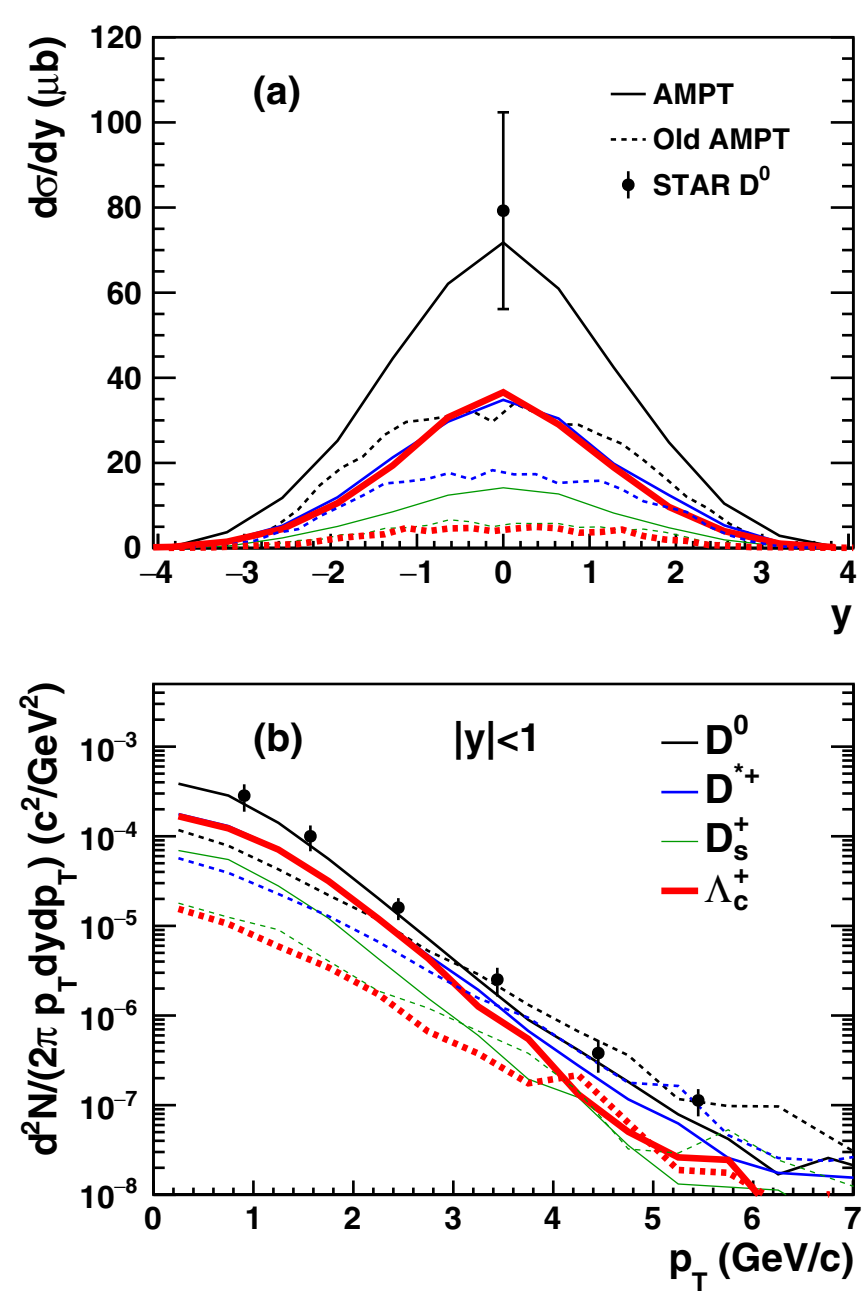

FIG. 4. (a) Rapidity distributions and (b) transverse momentum spectra of open charm hadrons in $p p$ collisions at $\sqrt{s}=200 \mathrm{GeV}$ from AMPT in comparison with the experimental data $[51,56]$.

quark production is subject to the minimum $p_{0}$ requirement, where we see much lower charm quark cross sections.

We show the rapidity and transverse momentum distributions of charm quarks from the AMPT model at $\sqrt{s}=200 \mathrm{GeV}$ and $\sqrt{s}=7 \mathrm{TeV}$ in Fig. 3. We see a significant enhancement of the charm quark yield in the updated AMPT compared to the old AMPT over all rapidities. The charm quark transverse momentum spectra at mid-rapidity are shown in Fig. 3(b), where the statistical errors of the AMPT results are represented with a shaded band. We also see that the removal of the transverse momentum cutoff $p_{0}$ for charm quarks mostly enhances the charm quark production in the low- $p_{\mathrm{T}}$ region. It is interesting to observe in Fig. 3 that the old AMPT result (dotted line) is rather similar to the result from the updated AMPT model with the $p_{0}$ cut for charm quarks (dashed curve); this is also seen in Fig. 2 until $1 \mathrm{TeV}$. Note that the old AMPT model also has a $p_{0}$ cut $(2 \mathrm{GeV} / c)$ on the charm quark production.

The productions of open charm hadrons in $p p$ collisions at $\sqrt{s}=200 \mathrm{GeV}$ are shown in Fig. 4 for $D^{0}, D^{*+}, D_{s}^{+}$, and $\Lambda_{c}^{+}$. Note that the charm quark or hadron yields and spectra
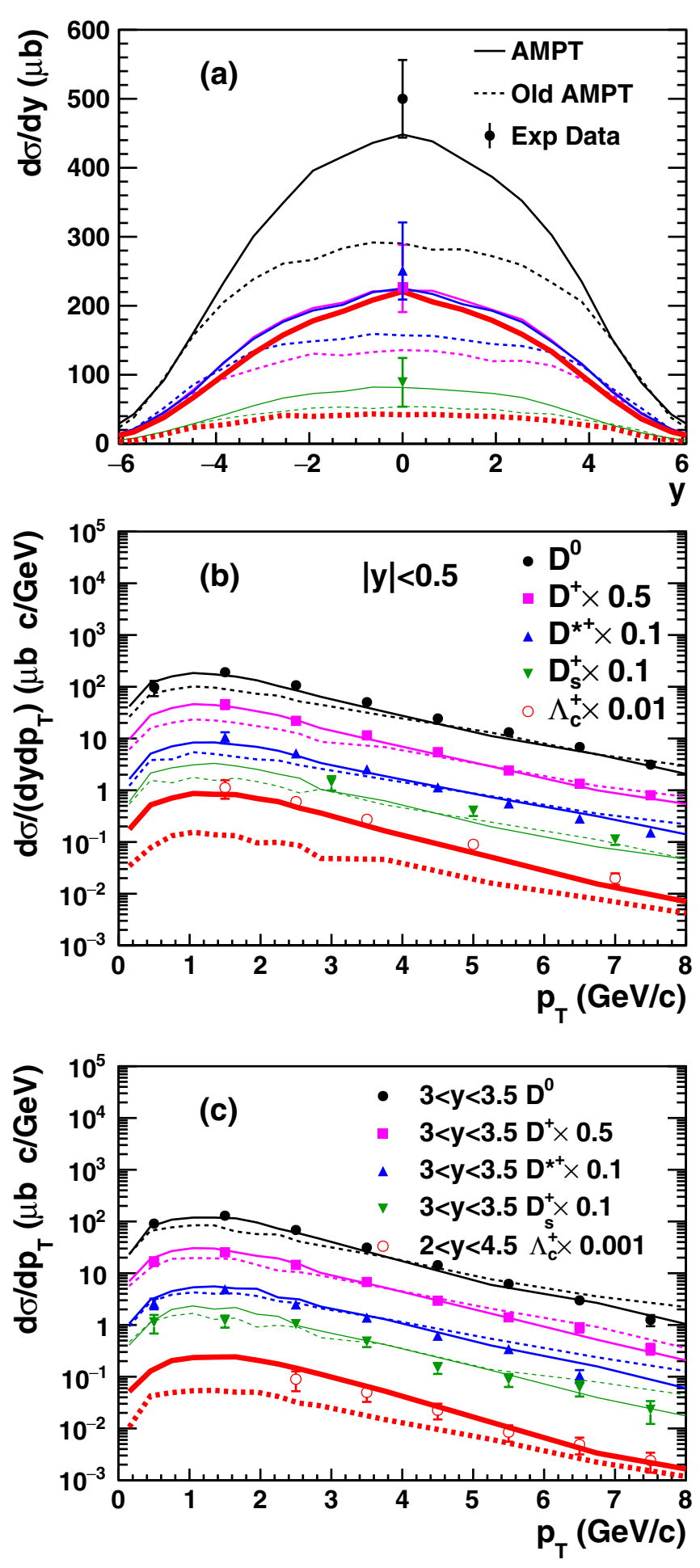

FIG. 5. (a) Rapidity distributions and (b) mid-rapidity transverse momentum spectra of open charm hadrons in $p p$ collisions at $\sqrt{s}=$ $7 \mathrm{TeV}$ from AMPT in comparison with the ALICE $D$ meson data [55] and $\Lambda_{c}$ data [57]. (c) Transverse momentum spectra of open charm hadrons in $p p$ collisions at $\sqrt{s}=7 \mathrm{TeV}$ from AMPT in comparison with the LHCb data at forward rapidity [52].

in this study have been averaged over those for particles and their corresponding antiparticles; e.g., the $\Lambda_{c}^{+}$results in Fig. 4 represent the average results for $\Lambda_{c}$ and $\bar{\Lambda}_{c}$. In addition, 
results of $D^{0}$ include both primordial $D^{0}$ mesons and $D^{0}$ mesons from $D^{*}$ decays. We see that the shape and magnitude of the charm hadron distributions shown in Fig. 4 reflect those of the charm quarks in Fig. 3. For example, the $p_{\mathrm{T}}$ spectra of charm hadrons from the updated AMPT model are much softer than those in the old AMPT model, as also seen for the charm quarks. The $D^{*}$ to $D^{0}$ ratio is rather flat across the shown $p_{\mathrm{T}}$ region. We also see that results from the updated AMPT model are slightly lower than the STAR $D^{0}$ data but still within the uncertainties.

In Fig. 5 we confront the model results for $D^{0}, D^{+}, D^{*+}$, $D_{s}^{+}$, and $\Lambda_{c}^{+}$with the experimental data for $p p$ collisions at $\sqrt{s}=7 \mathrm{TeV}$. Figure 5(a) shows that the results from the updated AMPT model for all these charm meson species roughly agree with the experimental data with large error bars, which mainly come from the extrapolation to the unmeasured low- $p_{\mathrm{T}}$ region in the experiment. Note that the charm hadron yields for all species in the updated AMPT model are much higher than those in the old AMPT. Figures 5(b) and 5(c) show the transverse momentum distributions of these charm hadrons at mid-rapidity and at forward rapidity, respectively, together with the experimental data. The updated AMPT model generally describes the data for different charm meson species $D^{0}, D^{+}, D^{*+}$, and $D_{s}^{+}$. The $\Lambda_{c}$ results are also roughly consistent with the experimental data (after our tuning of the coalescence parameter $r_{B M}=1$ for heavy hadrons), including the $\Lambda_{c}$ spectrum at forward rapidity.

\section{OPEN CHARM RESULTS FOR $A A$ COLLISIONS}

In $A A$ collisions, the charm production is subject to additional initial state and final state effects. Initial state effects include the nuclear modification to the parton distribution functions, while final state effects include parton rescattering in the ZPC parton cascade. We focus on central $A A$ collisions. Note that for hadron level results the event centrality class is determined according to the charged particle multiplicity

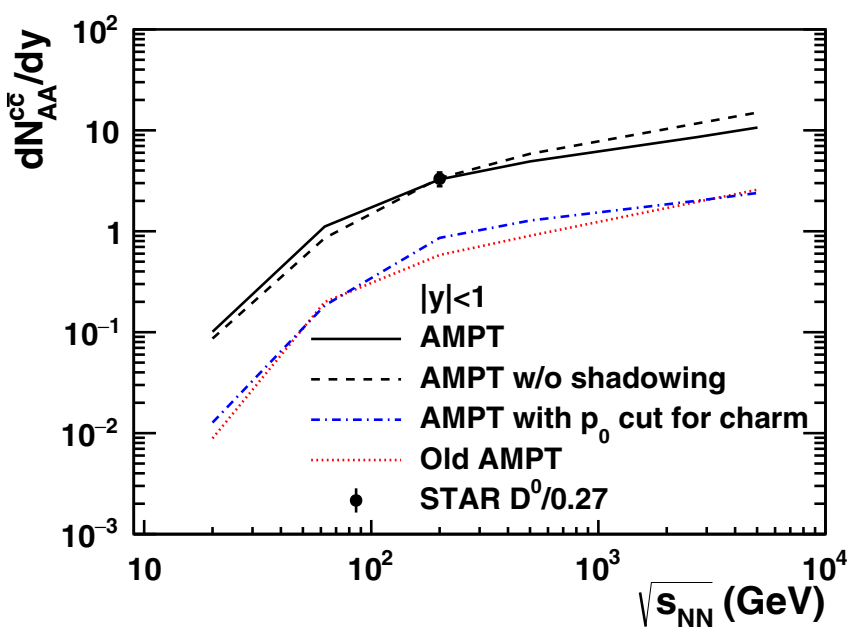

FIG. 6. Yields of charm quark pairs around mid-rapidity from AMPT for $0-10 \%$ central $\mathrm{Au}+\mathrm{Au}$ collisions at RHIC energies and $\mathrm{Pb}+\mathrm{Pb}$ collisions above RHIC energies as functions of the colliding energy in comparison with the extracted STAR data [56]. distribution generated by AMPT in the same way as done by the STAR (within $|\eta|<0.5$ ) [56] or ALICE (within $-3.7<$ $\eta<-1.7$ and $2.8<\eta<5.1$ ) experiment [58]. For the parton level results, the event centrality is obtained according to the impact parameter, where $b<4.88 \mathrm{fm}$ for $\mathrm{Au}+\mathrm{Au}$ collisions and $b<5.23 \mathrm{fm}$ for $\mathrm{Pb}+\mathrm{Pb}$ collisions is used to sample the $0-10 \%$ centrality events. We find that these two event centrality determination techniques give very similar results in central $A A$ collisions such as those at $0-10 \%$ centrality.

In Fig. 6, we show the charm quark yield at mid-rapidity for $0-10 \%$ central $A A$ collisions at different colliding energies. We see that the EPS09s nuclear modification slightly enhances the charm yield at low energies but significantly suppresses the charm yield at high energies (above $1 \mathrm{TeV}$ ). This results from the antishadowing effect at large $x$ and the shadowing effect at small $x$. We also see that the AMPT result agrees with the charm quark pair yield for $0-10 \%$ central $\mathrm{Au}+\mathrm{Au}$ collisions at $\sqrt{s_{N N}}=200 \mathrm{GeV}$ extracted from the STAR $D^{0}$ data [56]. The $D^{0}$ fragmentation fraction 0.27 is estimated based on the STAR data of $d \sigma\left(D^{0}\right) / d y=41 \mu \mathrm{b}$ and
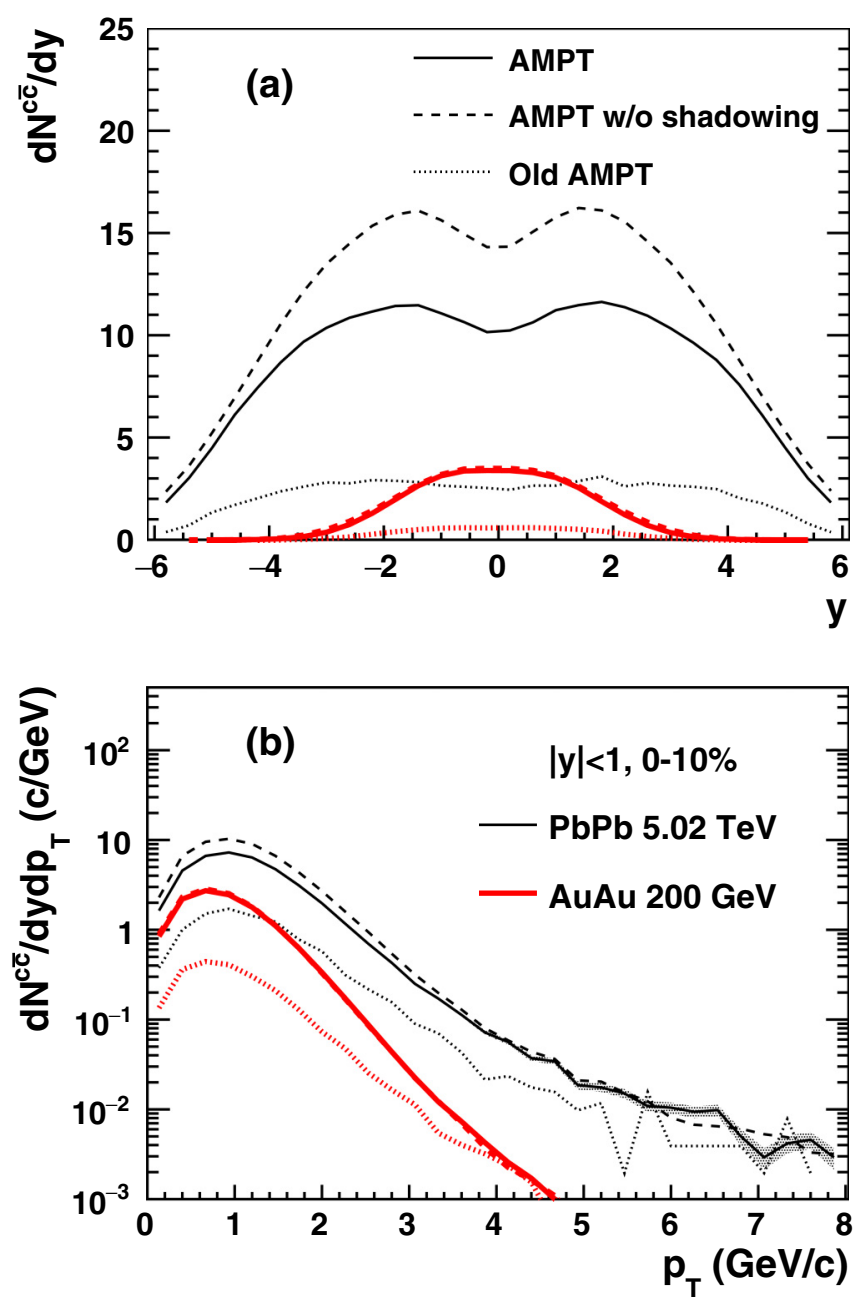

FIG. 7. (a) Rapidity distributions and (b) transverse momentum spectra of charm quarks in central $\mathrm{Au}+\mathrm{Au}$ collisions at $\sqrt{s_{N N}}=$ $200 \mathrm{GeV}$ (thick) and central $\mathrm{Pb}+\mathrm{Pb}$ collisions at $\sqrt{s_{N N}}=5.02 \mathrm{TeV}$ (thin) from the AMPT model. 
$d \sigma\left(D^{0}+D^{+}+D_{s}^{+}+\Lambda_{c}^{+}\right) / d y=152 \mu \mathrm{b}$ for $10-40 \% \mathrm{Au}+$ $\mathrm{Au}$ collisions at $\sqrt{s_{N N}}=200 \mathrm{GeV}$ [59]. In contrast to an earlier extraction [60] where the same $c \rightarrow D^{0}$ fragmentation ratio $(0.565)$ as in $p+p$ collisions was assumed, the current $c \rightarrow D^{0}$ fraction takes into account the $\Lambda_{c}$ and $D_{s}$ enhancements observed in the $\mathrm{Au}+\mathrm{Au}$ data. Compared to the old AMPT results (dotted line), the current AMPT results (solid line) for the charm quark yield at mid-rapidity are significantly higher (by a factor of $\approx 5$ ). The main reason for this enhancement is our removal of the $p_{0}$ cut for heavy flavor productions, as also shown by the dot-dashed curve. In addition, the EPS09s nuclear shadowing implemented in the updated AMPT model is generally weaker than the original parametrized shadowing function in HIJING 1.0.

We show the charm quark distributions in Fig. 7 for $\mathrm{Au}+$ $\mathrm{Au}$ collisions at $\sqrt{s_{N N}}=200 \mathrm{GeV}$ and $\mathrm{Pb}+\mathrm{Pb}$ collisions at $\sqrt{s_{N N}}=2.76 \mathrm{TeV}$ for $0-10 \%$ centrality. We see in Fig. 7(a) that the updated AMPT model (solid line) gives significantly more charm quarks compared to the old AMPT model (dotted line) at both energies, which is already shown in Fig. 6. On the
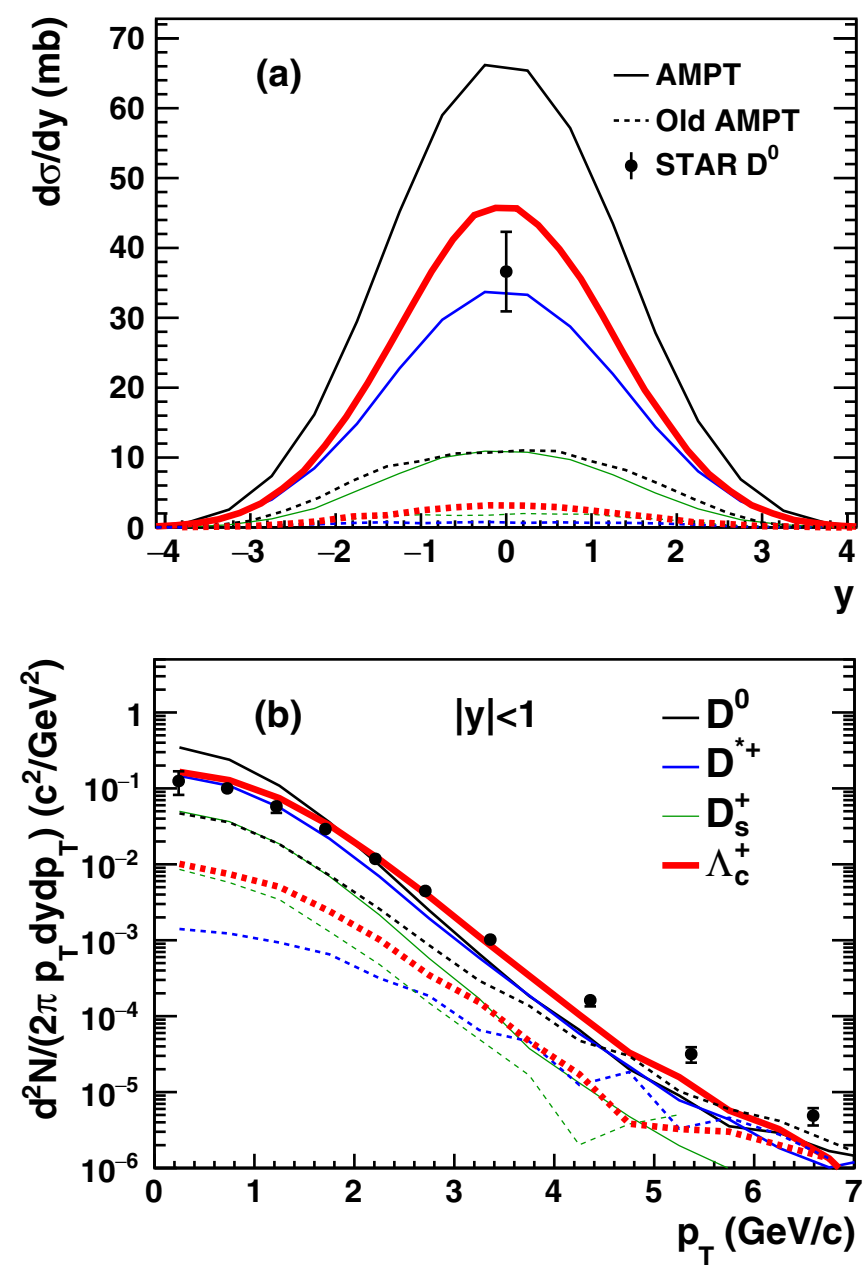

FIG. 8. (a) Rapidity distributions and (b) transverse momentum spectra of open charm hadrons in $0-10 \%$ central $\mathrm{Au}+\mathrm{Au}$ collisions at $\sqrt{s_{N N}}=200 \mathrm{GeV}$ from AMPT in comparison with the STAR $D^{0}$ data [56]. other hand, nuclear shadowing leads to a $\approx 50 \%$ suppression of the rapidity density of charm quarks for central $\mathrm{Pb}+\mathrm{Pb}$ collisions at $\sqrt{s_{N N}}=5.02 \mathrm{TeV}$. We can find in Fig. 7(b) that nuclear shadowing mainly suppresses the charm quark yield in the low- $p_{\mathrm{T}}$ region. This is consistent with the fact that nuclear shadowing is stronger at low $\mu_{F}^{2}$ that is associated with low- $p_{\mathrm{T}}$ charm quarks. Note that the EPS09s nuclear shadowing functions in the updated AMPT model include the QCD evolution with the $\mu_{F}$, unlike the shadowing parametrization implemented in HIJING 1.0 as well as in the old AMPT model.

The productions of open charm hadrons including $D^{0}, D^{*+}$, $D_{s}^{+}$and $\Lambda_{c}$ in $0-10 \%$ central $\mathrm{Au}+\mathrm{Au}$ collisions at $\sqrt{s_{N N}}=$ $200 \mathrm{GeV}$ are shown in Fig. 8. As shown in Fig. 8(a), unlike the small $D^{*+} / D^{0}$ ratio in the old AMPT model, the $D^{0}$ yield from the updated AMPT model is about twice the $D^{*+}$ yield, close to the ratio observed in Fig. 4(a) for $p p$ collisions at the same energy. Compared to the mid-rapidity STAR $D^{0}$ data, however, the AMPT result is significantly higher. Also, the AMPT result on the $D^{0} p_{\text {T }}$ spectrum in Fig. 8(b) is too soft in comparison with the STAR data $[56,60]$ and underpredicts the
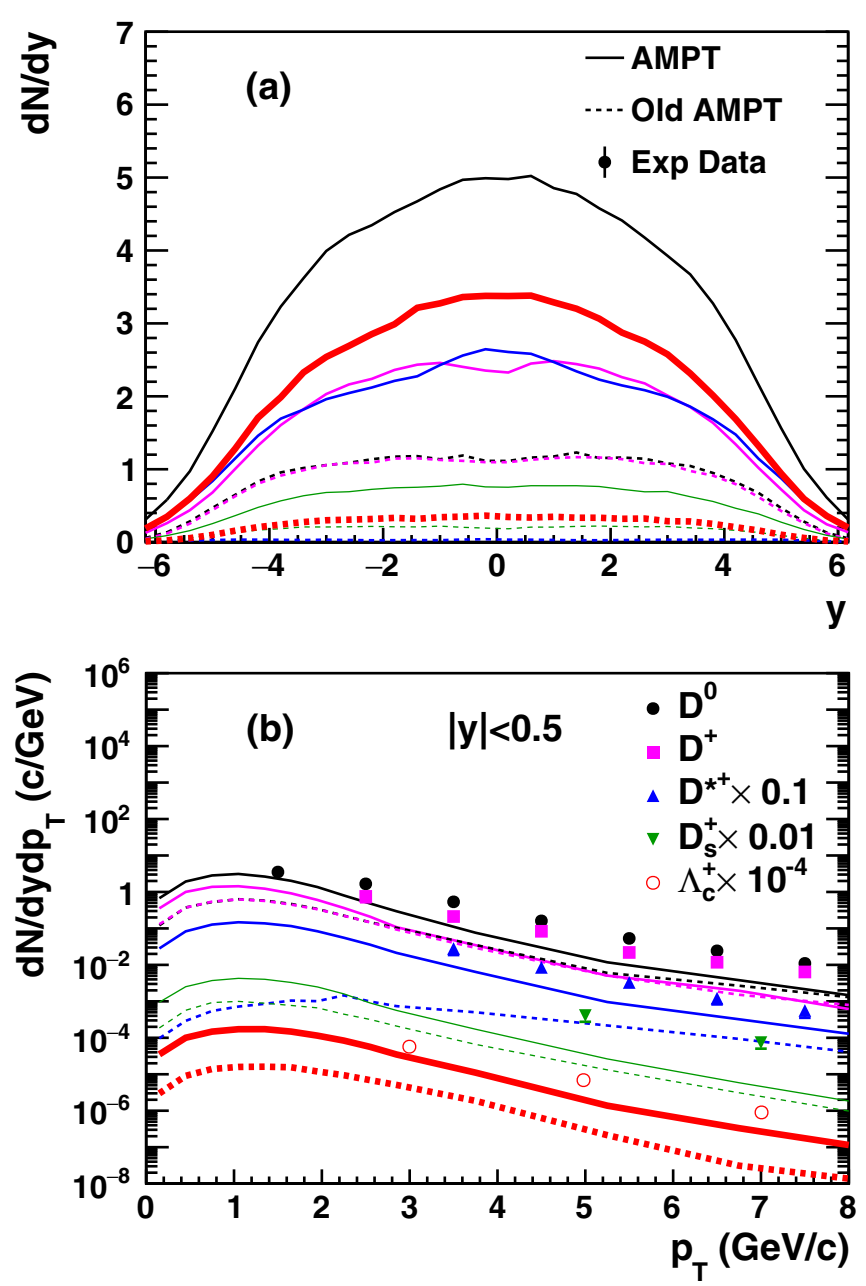

FIG. 9. (a) Rapidity distributions and (b) transverse momentum spectra of open charm hadrons in $0-10 \%$ central $\mathrm{Pb}+\mathrm{Pb}$ collisions at $\sqrt{s_{N N}}=5.02 \mathrm{TeV}$ from AMPT in comparison with the ALICE data $[58,62]$. 
$D^{0}$ production at $p_{\mathrm{T}}>4 \mathrm{GeV} / c$. Since the yield of midrapidity charm quarks from AMPT is consistent with the extracted STAR data as shown in Fig. 6, the overestimation of the $D^{0}$ yield in AMPT could be because the quark coalescence in AMPT gives fewer $D_{s}$ and $\Lambda_{c}$ than the data [59]. It has been suggested that a sequential coalescence of different charm hadrons is important for the enhancement of $\Lambda_{c} / D^{0}$ and $D_{s} / D^{0}$ ratios in $A A$ collisions [61]. Including this sequential coalescence picture into AMPT could improve the descriptions of different charm hadron species in the future. In addition, the charm $p_{\mathrm{T}}$ spectra depend on the scatterings' cross section and its angular distribution between charm quarks and light flavors. The AMPT model currently uses the $g+g \rightarrow g+g$ cross section for scatterings between all parton flavors, and improvements should be made to treat parton scatterings between different flavors differently, where the comparison with the charm $p_{\mathrm{T}}$ spectra data will enable us to extract the charm interaction strength with light flavors.

In Fig. 9 we show open charm hadrons in $0-10 \%$ central $\mathrm{Pb}+\mathrm{Pb}$ collisions at $\sqrt{s_{N N}}=5.02 \mathrm{TeV}$ and compare with the ALICE data. We first see that the model results for all these open charm particles are lower than the experimental data, especially in the higher $p_{\mathrm{T}}$ region. When we integrate the midrapidity $D^{0}$ yield at $p_{\mathrm{T}} \geqslant 1.0 \mathrm{GeV} / c$, the ALICE data give 6.0 while the AMPT result gives 3.3. This underestimation of the $D^{0}$ yield in AMPT is first related to the branching of charm quarks into different hadron species; e.g., the $\Lambda_{c} / D^{0}$ ratio from AMPT is higher than the LHC data as shown in Fig. 10(d). Second, the $D^{0} p_{\mathrm{T}}$ spectrum from the AMPT model is too soft, as also seen at RHIC energies in Fig. 8(b). Furthermore, the total charm quark yield in AMPT could be lower than that in the ALICE data, in part because of nuclear shadowing that has been shown in Fig. 6 to significantly suppresses the charm yield at LHC energies. Note that there is still a large uncertainty on the nuclear shadowing of gluons [47], which we have not explored in this study.

We examine in Fig. 10 the ratios of open charm hadron yields in $7 \mathrm{TeV} p p$ collisions and $0-10 \%$ central $\mathrm{Pb}+\mathrm{Pb}$ collisions at 5.02 TeV. Note that, although the AMPT results of the charm hadron yields are significantly lower than the experimental data for $\mathrm{Pb}+\mathrm{Pb}$ collisions at $\mathrm{LHC}$, the ratios of different charm species are often compatible with the data. It has been discussed that the $D_{s}^{+}$production in large collision systems may be enhanced due to the strangeness enhancement, as the data in Fig. 10(c) may suggest. The AMPT results show no significant change in the strange-to-nonstrange $D$ meson ratio in $A A$ collisions; however, sequential coalescence of different charm species [61] could change these results.

Regarding the $\Lambda_{c}^{+} / D^{0}$ ratio shown in Fig. 10(d), the results from AMPT are consistent with data in $p p$ collisions and show an enhancement in the intermediate $p_{\mathrm{T}}$ region in $A A$ collisions. However, the current ALICE data $[55,57,58,62]$ seem to favor a rather weak enhancement of charm baryons over charm mesons. We also show the AMPT result for the
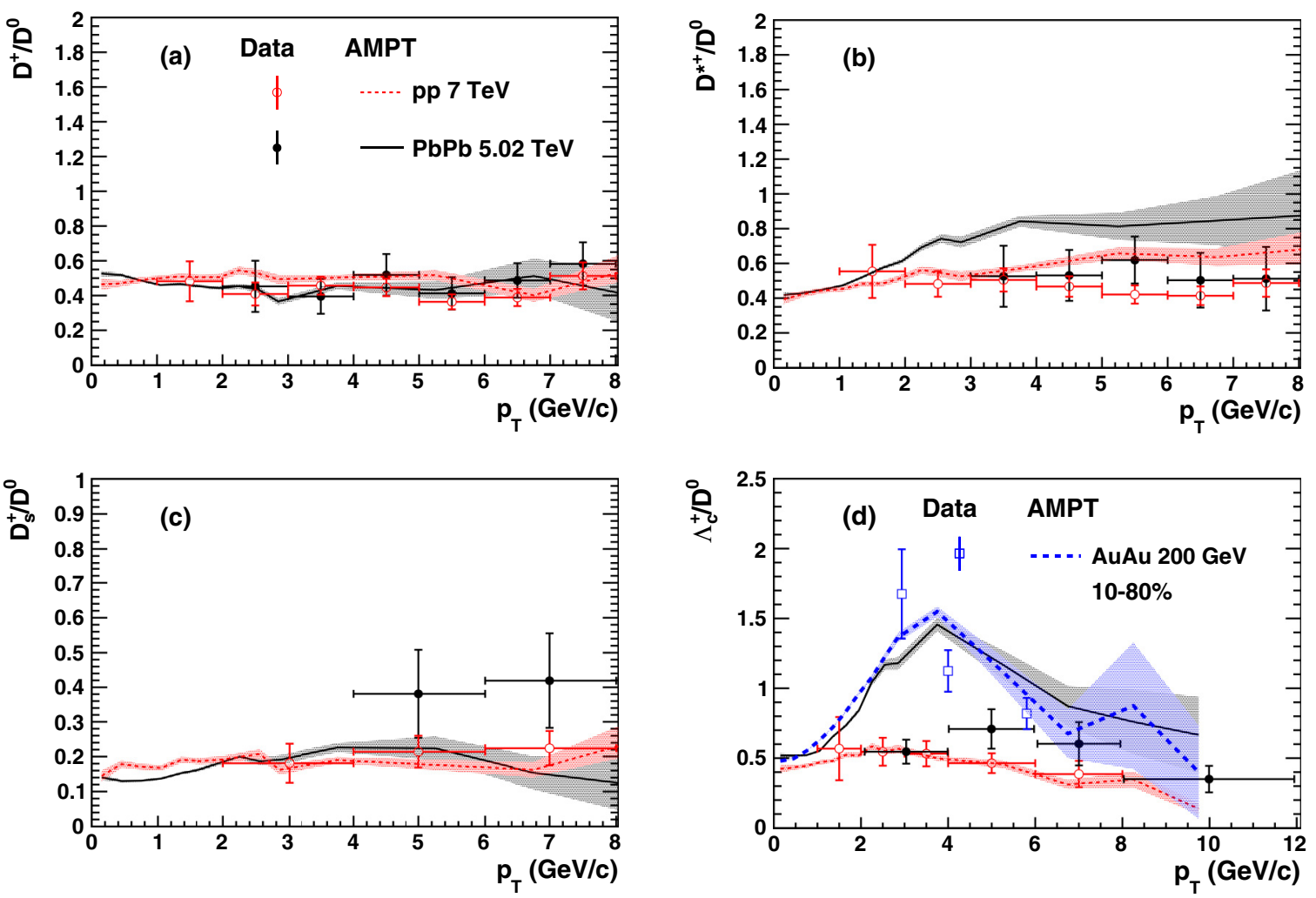

FIG. 10. Ratios of mid-rapidity open charm hadron yields as functions of $p_{\mathrm{T}}$ in $p p$ collisions at $\sqrt{s}=7 \mathrm{TeV}$ and $0-10 \%$ central $\mathrm{Pb}+\mathrm{Pb}$ collisions at $\sqrt{s_{N N}}=5.02 \mathrm{TeV}$ from the AMPT model (curves) in comparison with the experimental data $[55,57,58,62]:$ (a) $D^{+} / D^{0}$, (b) $D^{*+} / D^{0}$, (c) $D_{s}^{+} / D^{0}$, and (d) $\Lambda_{c}^{+} / D^{0}$. Panel (d) also shows the AMPT result for 10-80\% central Au $+\mathrm{Au}$ collisions at $200 \mathrm{GeV}$ in comparison with the STAR data [59]. 
10-80\% centrality of $\mathrm{Au}+\mathrm{Au}$ collisions at $200 \mathrm{GeV}$, which shows a significant $\Lambda_{c}^{+} / D^{0}$ enhancement in rough agreement with the STAR data [59].

\section{DISCUSSIONS}

In the string melting AMPT model, the interactions between charm quarks and the QGP medium are modeled by parton elastic scatterings of ZPC. Thus our study includes the collisional energy loss of heavy quarks but neglects the radiative energy loss. Studies have suggested that the elastic collisional energy loss is dominant for heavy flavors below a moderately high $p_{\mathrm{T}}$, e.g., for charm hadrons at $p_{\mathrm{T}} \lesssim 5-6 \mathrm{GeV} / c$ in $\mathrm{Au}+$ $\mathrm{Au}$ collisions at $\sqrt{s_{N N}}=200 \mathrm{GeV}$ or at $p_{\mathrm{T}} \lesssim 15 \mathrm{GeV} / c$ in $\mathrm{Pb}+\mathrm{Pb}$ collisions at $\sqrt{s_{N N}}=2.76 \mathrm{TeV}$ [22]. Therefore results in our study are applicable from low to moderately high $p_{\mathrm{T}}$ but not at very high $p_{\mathrm{T}}$.

In the ZPC parton cascade, the parton cross section and its angular distribution determine the interaction strength between heavy quarks and the medium. Note that any given scattering angular distribution can be exactly sampled with no need of the assumption of small-angle scatterings; this is an advantage of the parton cascade approach. After the quark coalescence process, the formed hadrons go through hadron interactions as modeled by an extended ART model [25,63]. Currently we have not implemented any hadron interactions for heavy hadrons except for decays of the heavy hadron resonances.

It is also interesting to note that, while we have removed the $p_{0}$ cut for initial heavy flavor productions, we need to use a $p_{0}$ cut, which grows with the collision energy and the system size, for the initial light flavor minijet production to describe charged particle as well as light flavor multiplicities in heavy ion collisions at high energies [36]. This different treatment of the $p_{0}$ cut for different flavors in the final state seems to be inconsistent with initial state saturation models but might be understandable within final state saturation models such as the EKRT model [64].

\section{SUMMARY}

In this work, we use the recently updated AMPT model to study open heavy flavor productions. In addition to the incorporation of modern parton distribution functions in nuclei, we have removed the transverse momentum cutoff $p_{0}$ for the pQCD heavy flavor production channels. Systematic comparisons to the experimental data show that the updated AMPT model can well describe the yields and $p_{\mathrm{T}}$ spectra of open charm hadrons including $D, D^{*}, D_{s}$, and $\Lambda_{c}$ in $p p$ collisions at different energies. The updated model also describes the charm data in central $A A$ collisions much better than the previous AMPT model, although it gives softer charm hadron spectra than the experimental data and also underestimates the open charm hadron yields in central $\mathrm{Pb}+\mathrm{Pb}$ collisions at LHC energies. These improvements in the AMPT model lay a foundation for further studies of heavy flavor observables together with light flavor observables within the transport model framework.

\section{ACKNOWLEDGMENTS}

We thank Xinye Peng for helpful discussions. This work is supported by National Natural Science Foundation of China under Grants No. 11890711 and No. 11905188 and by the Fundamental Research Funds for the Central Universities, China University of Geosciences (Wuhan) under Grant No. CUG180615.
[1] A. Andronic et al., Eur. Phys. J. C 76, 107 (2016).

[2] B. Muller and X.-N. Wang, Phys. Rev. Lett. 68, 2437 (1992).

[3] Z.-W. Lin and M. Gyulassy, Phys. Rev. C 51, 2177 (1995); 52, 440(E) (1995).

[4] S. K. Das, S. Plumari, S. Chatterjee, J. Alam, F. Scardina, and V. Greco, Phys. Lett. B 768, 260 (2017).

[5] S. Chatterjee and P. Bożek, Phys. Rev. Lett. 120, 192301 (2018).

[6] M. Nasim and S. Singha, Phys. Rev. C 97, 064917 (2018).

[7] M. He, R. J. Fries, and R. Rapp, Phys. Rev. Lett. 110, 112301 (2013).

[8] K. Huggins and R. Rapp, Nucl. Phys. A 896, 24 (2012).

[9] T. Lang, H. van Hees, G. Inghirami, J. Steinheimer, and M. Bleicher, Phys. Rev. C 93, 014901 (2016).

[10] M. L. Mangano, P. Nason, and G. Ridolfi, Nucl. Phys. B 373, 295 (1992).

[11] B. A. Kniehl, G. Kramer, I. Schienbein, and H. Spiesberger, Phys. Rev. D 71, 014018 (2005).

[12] I. Helenius and H. Paukkunen, J. High Energy Phys. 05 (2018) 196.

[13] M. Cacciari, P. Nason, and R. Vogt, Phys. Rev. Lett. 95, 122001 (2005).

[14] M. Djordjevic and M. Djordjevic, Phys. Rev. C 92, 024918 (2015).
[15] J. Xu, J. Liao, and M. Gyulassy, J. High Energy Phys. 02 (2016) 169.

[16] A. Beraudo, A. De Pace, M. Monteno, M. Nardi, and F. Prino, Eur. Phys. J. C 75, 121 (2015).

[17] M. He, R. J. Fries, and R. Rapp, Phys. Lett. B 735, 445 (2014)

[18] S. Cao, G.-Y. Qin, and S. A. Bass, Phys. Rev. C 92, 024907 (2015).

[19] J. Uphoff, O. Fochler, Z. Xu, and C. Greiner, J. Phys. G 42, 115106 (2015).

[20] S. Cao, T. Luo, G.-Y. Qin, and X.-N. Wang, Phys. Lett. B 777, 255 (2018).

[21] M. Nahrgang, J. Aichelin, P. B. Gossiaux, and K. Werner, Phys. Rev. C 89, 014905 (2014).

[22] T. Song, H. Berrehrah, D. Cabrera, W. Cassing, and E. Bratkovskaya, Phys. Rev. C 93, 034906 (2016).

[23] S. K. Das, F. Scardina, S. Plumari, and V. Greco, Phys. Lett. B 747, 260 (2015).

[24] S. Plumari, V. Minissale, S. K. Das, G. Coci, and V. Greco, Eur. Phys. J. C 78, 348 (2018).

[25] Z.-W. Lin, C. M. Ko, B.-A. Li, B. Zhang, and S. Pal, Phys. Rev. C 72, 064901 (2005).

[26] B. Zhang, Comput. Phys. Commun. 109, 193 (1998). 
[27] B. Zhang, L.-W. Chen, and C.-M. Ko, Phys. Rev. C 72, 024906 (2005).

[28] H. Li, Z.-W. Lin, and F. Wang, Phys. Rev. C 99, 044911 (2019).

[29] L. He, T. Edmonds, Z.-W. Lin, F. Liu, D. Molnar, and F. Wang, Phys. Lett. B 753, 506 (2016).

[30] Z.-W. Lin, L. He, T. Edmonds, F. Liu, D. Molnar, and F. Wang, Nucl. Phys. A 956, 316 (2016).

[31] A. Kurkela, A. Mazeliauskas, J.-F. Paquet, S. Schlichting, and D. Teaney, Phys. Rev. Lett. 122, 122302 (2019).

[32] A. Kurkela, A. Mazeliauskas, J.-F. Paquet, S. Schlichting, and D. Teaney, Phys. Rev. C 99, 034910 (2019).

[33] S. Cao et al., Phys. Rev. C 99, 054907 (2019).

[34] Y. Xu et al., Phys. Rev. C 99, 014902 (2019).

[35] Y. He and Z.-W. Lin, Phys. Rev. C 96, 014910 (2017).

[36] C. Zhang, L. Zheng, F. Liu, S. Shi, and Z.-W. Lin, Phys. Rev. C 99, 064906 (2019).

[37] M. Gyulassy and X.-N. Wang, Comput. Phys. Commun. 83, 307 (1994).

[38] T. Sjostrand, S. Mrenna, and P. Z. Skands, J. High Energy Phys. 05 (2006) 026.

[39] M. Bahr et al., Eur. Phys. J. C 58, 639 (2008).

[40] E. Norrbin and T. Sjostrand, Eur. Phys. J. C 17, 137 (2000).

[41] X.-N. Wang and M. Gyulassy, Phys. Rev. D 44, 3501 (1991).

[42] W.-T. Deng, X.-N. Wang, and R. Xu, Phys. Rev. C 83, 014915 (2011).

[43] Z.-W. Lin and C. M. Ko, Phys. Rev. C 65, 034904 (2002).

[44] S. Singha, B. Mohanty, and Z.-W. Lin, Int. J. Mod. Phys. E 24, 1550041 (2015).

[45] M. He and R. Rapp, Phys. Lett. B 795, 117 (2019).

[46] D. Stump, J. Huston, J. Pumplin, W.-K. Tung, H. L. Lai, S. Kuhlmann, and J. F. Owens, J. High Energy Phys. 10 (2003) 046.
[47] I. Helenius, K. J. Eskola, H. Honkanen, and C. A. Salgado, J. High Energy Phys. 07 (2012) 073.

[48] Z.-W. Lin, Phys. Rev. C 90, 014904 (2014).

[49] C. Lourenco and H. K. Wohri, Phys. Rep. 433, 127 (2006).

[50] A. Adare et al. (PHENIX Collaboration), Phys. Rev. C 84, 044905 (2011).

[51] L. Adamczyk et al. (STAR Collaboration), Phys. Rev. D 86, 072013 (2012).

[52] R. Aaij et al. (LHCb Collaboration), Nucl. Phys. B 871, 1 (2013).

[53] G. Aad et al. (ATLAS Collaboration), Nucl. Phys. B 907, 717 (2016).

[54] B. Abelev et al. (ALICE Collaboration), J. High Energy Phys. 07 (2012) 191.

[55] S. Acharya et al. (ALICE Collaboration), Eur. Phys. J. C 77, 550 (2017).

[56] J. Adam et al. (STAR Collaboration), Phys. Rev. C 99, 034908 (2019).

[57] S. Acharya et al. (ALICE Collaboration), J. High Energy Phys. 04 (2018) 108.

[58] S. Acharya et al. (ALICE Collaboration), J. High Energy Phys. 10 (2018) 174.

[59] G. Xie (STAR Collaboration), PoS Hard Probes 2018, 142 (2018).

[60] L. Adamczyk et al. (STAR Collaboration), Phys. Rev. Lett. 113, 142301 (2014); 121, 229901(E) (2018).

[61] J. Zhao, S. Shi, N. Xu, and P. Zhuang, arXiv:1805.10858.

[62] C. Zampolli, presented at Strangeness in Quark Matter 2019, Bari, Italy, https://indico.cern.ch/event/755366/contributions/ 3396487/attachments/1859933/3056377/CZampolli_v7.pdf

[63] B.-A. Li and C. M. Ko, Phys. Rev. C 52, 2037 (1995).

[64] K. J. Eskola, K. Kajantie, P. V. Ruuskanen, and K. Tuominen, Nucl. Phys. B 570, 379 (2000). 